\title{
The Roots of the Theoretical Models of the Nanotechnoscience in the Electric Circuit Theory
}

\author{
Vitaly Gorokhov ${ }^{1,2}$ \\ ${ }^{1}$ Institute of Philosophy, Russian Academy of Sciences, Moscow, Russia \\ ${ }^{2}$ Institute of Technology Assessment and System Analysis, Karlsruhe Institute of Technology, \\ Karlsruhe, Germany \\ Email: vitaly.gorokhov@mail.ru,vitaly.gorokhov@kit.edu
}

Received April $8^{\text {th }}, 2013$; revised May $12^{\text {th }}, 2013$; accepted May $20^{\text {th }}, 2013$

\begin{abstract}
Copyright (C) 2013 Vitaly Gorokhov. This is an open access article distributed under the Creative Commons Attribution License, which permits unrestricted use, distribution, and reproduction in any medium, provided the original work is properly cited.
\end{abstract}

\begin{abstract}
In the contemporary nanotechnoscience makes natural-scientific experimentation constitutive for design, while research results are oriented equally on interpreting and predicting the course of natural processes, and on designing devices. Nanoystems can be seen as nanoelectrical switches in a nanocircuit. In nanocircuit structure, we find traditional electronic components at different levels, realized on the basis of nanotechnology. In nanotechnoscience explanatory models of natural phenomena are proposed, and predictions of the course of certain natural events on the basis of mathematics and experimental data are formulated, on the one hand, as in classical natural science; as in the engineering sciences, on the other hand, not only experimental setups, but also structural plans for new nanosystems previously unknown in nature and technology are devised. In nanotechnoscience different models (equivalent circuits with standard electronic components) of electric circuit theory are used for the analysis and synthesis of nanocircuits, and a special nanocircuit theory is elaborated. So nanotechnology is, at the same time, a field of scientific knowledge and a sphere of engineering activity - in other words, NanoTechnoScience, similar to Systems Engineering as the analysis and design of complex micro- and nanosystems.
\end{abstract}

Keywords: History of Science; History of Engineering Science; Nanotechnoscience; Electric Circuits Theory; Electronic Nanocircuit; Circuits Models of Nanosystems; Natural Science; Engineering Science; Science and Engineering

\section{Introduction}

Contemporary technoscience makes natural scientific experimentation inseparable from design, while research results are equally oriented to interpret and predict the course of natural processes and to design structures.

Engineering theory is oriented not toward interpreting and predicting the course of natural processes but toward designing engineering schemes. Natural scientific knowledge and laws must be considerably specified and modified in engineering theory to be applicable to practical engineering problems. To adapt theoretical knowledge to the level of practical engineering recommendations, technical theory develops special rules that establish a correspondence between the abstract objects of engineering theory and the structural components of real engineering systems and operations that transfer theoretical results into engineering practice. Engineering sciences are specific because their engineering practice replaces experiments, as a rule. It is engineering activity that checks the adequacy of theoretical engineering conclusions and serves as a source of new empirical knowledge.

In the nanotechnoscience is equal important the explanation and prognostication of the course natural processes (like in natural science) and multiplying of structural schemes of nanosystems (like in engineering science). Electron beam lithogra- phy system is at the same time experimental investigation system and is used for the nanofabrication as so-called "nanowriter"

It is well-known that, in nanotechnoscience, constructs from various scientific theories-classical and quantum physics, classical and quantum chemistry, structural biology, etc.- -are used, whereas, in nanosystems, different physical, chemical and biological processes take place. However one can also construct the circuit on the basis of definite nanosructures, such as, e.g., a super-heterodyne radio receiver on the nanolevel (see: Bhushan 2004: p. 240).

In the nanotechnoscience for analysis and synthesis of the nanocircuits also are used the different models (equivalent circuits with standard electronics components) of the electric circuit theory and is elaborated a special nanocircuit theory. In the structure of the nanocircuits we can find many different traditional electronic components ("molecular-scale electronics") realized on the nanolevel with the help of nanotechnology: first, there are electronic elements, second, electronics blocks, and third, large-scale nanosystems.

\section{The Structure of NanoTechnoScience}

In nanotechnoscience, on the one hand, explanatory models of natural phenomena are drawn up and predictions of the 
course of certain natural events on the basis of mathematics and experimental data are formulated as in classical natural science, and, as in the engineering sciences on the other hand, not only experimental arrangements are constructed, but also structural plans of new nanosystems previously unknown in nature and technology (Figure 1).

Three main levels in the theoretical (ontological) schemes of a nano scientific theory can be discerned, namely mathematically oriented functional schemes, "flow" schemes reflecting natural processes going in the investigated or constructed system, and structural schemes representing its structural parameters and engineering analysis, i.e. systems structure.

The functional scheme is oriented on the mathematical description and fixes the general idea about the system (for example, nanosystem), irrespective of the method of its realization. The units of this scheme reflect only the functional properties of the elements of the system for the sake of which they are included in it to attain the general objective and reflect certain mathematical relations. The blocks of this scheme reflect only those functional properties of the systems elements, for which they are incorporated and which contribute to achieving the common purpose. The blocks express generalized mathematical operations and their relations are particular mathematical dependences. But they can be expressed as a simple decomposition of interrelated functions aimed at achieving the customerprescribed common purpose of system under investigation and/ or design. Such a functional scheme is used to construct a system algorithm and determine a system configuration.

Flow schemes (for example, flow block diagram) describe natural, for instance, physical processes taking place in the technical system and connecting its elements into a single whole. The units of such schemes reflect various operations performed in the natural process by the elements of the technical system while it is functioning. These are based on natural- scientific concepts first of all physical processes.

In the nanotechnology they present not only physical (electrical, mechanical, hydraulic, etc.) processes, but also chemical and biological ones, that is to say any natural processes in general. The blocks of these schemes reflect various operations performed by the elements of the nanotechnological system during its function. In the extreme general terms, "flow" schemes represent not only natural processes, but also any flow of "substance" (matter, energy or information).

Structural schemes reflect the structural arrangement of elements and linkages in the given system and presuppose its possible realization. They are the theoretical drafts of the systems structure to elaborate a project of the experimental situation together with the experimental equipment. Hertz for example developed structural schemes and a conceptual apparatus corresponding to them - such concepts as the dipole and vibrator. The scrupulous description of test equipment designs (e.g., of mirror material, shape and dimensions, etc.) was combined with the general description of experimental measurement situations, the latter being a prototype of future electric circuits of the radio receiver and radio transmitter. In the nanotechnology can be another realization as in the traditional electronics but the structural scheme is similar. For example, the one of the main elements of electric circuits-capacitor can receive in nantotechnology another construction as conventional Faraday capacitor but has the similar representation as two-terminal network-capacitive resistance.

The structural scheme gives nodal points of "flows" (operating processes) which can be equipment items, parts of or even entire complicated systems. The elements of the latter are regarded in them as having not only functional properties, but also properties of the second order, i.e. those undesirable properties which are added by a definitely realized element, for instance, non-linear distortions of the amplified signal in the

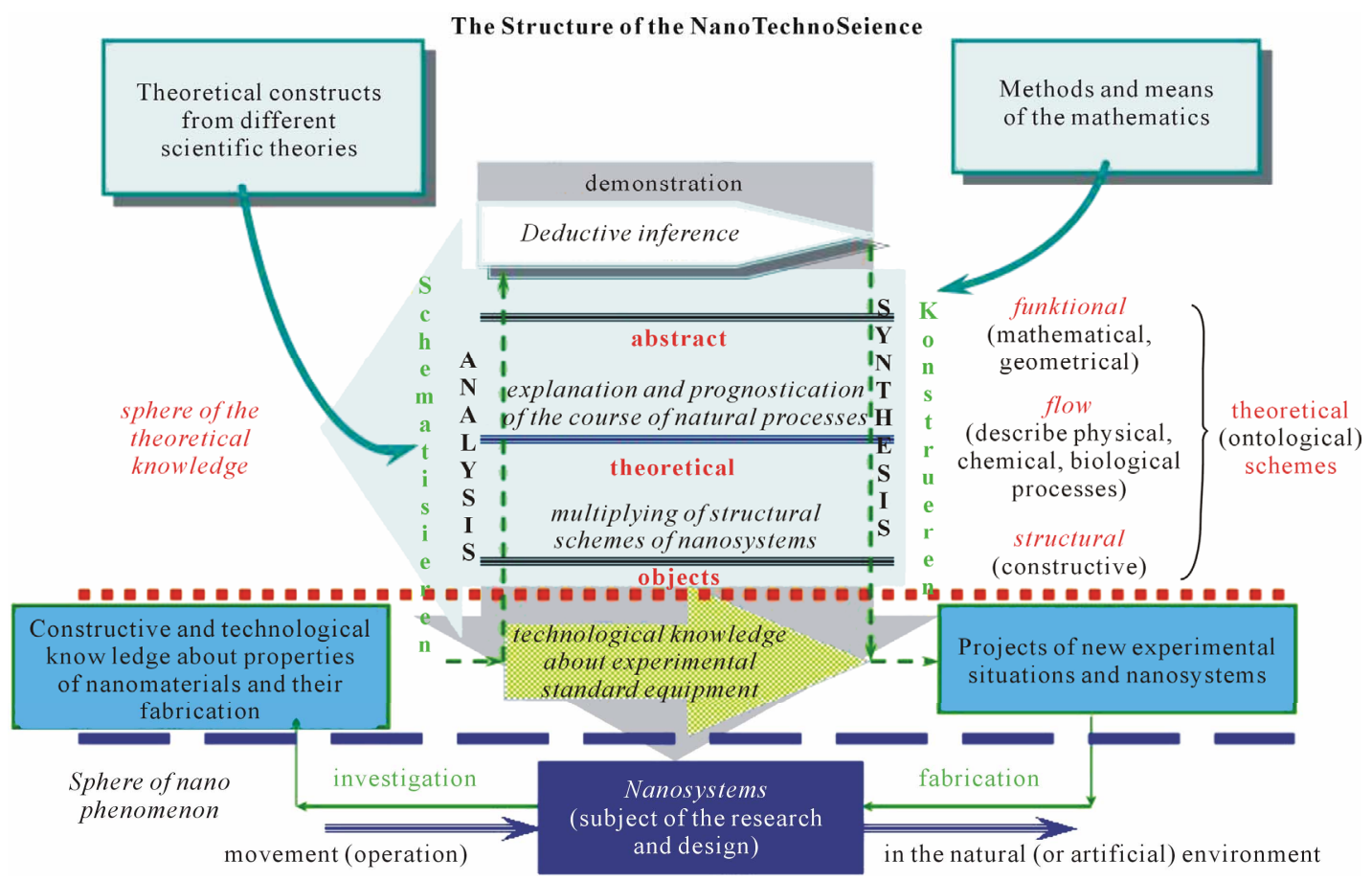

Figure 1.

The structure of NanoTecnoScience. 
amplifier. These schemes represent constructive-technical and technological parameters, i.e. they reflect specific problems cropping up in engineering practice. In modern man-machine and nanobio hybrid systems, such a realization can be of diverse types and even be a non-engineering and non-physical one. Therefore, the terms "technical parameters", "construction" are not apt here. The case in point is the configuration of systems, their general structure.

From the radio electronics point of view it makes no difference what kind of the realization has the circuit (also as nanostructure). His blocs and elements can be represented in all cases as the correspondent equivalent circuits with standard electronics components.

Let us consider the specific features of the above-mentioned theoretical schemes of engineering science, referring to the electric circuit theory.

Even structural schemes of electric engineering are idealizations of real electric circuits. They omit many of particular characteristics of an electrical device, such as its overall dimensions, weight, assembly techniques, etc. (they are specified during design work and manufacture, i.e. during engineering itself). Such schemes give general structural and technical, and manufacturing parameters of standardized structural elements (resistors, inductance coils, batteries, etc.), which will be used in further analysis, namely, their types and dimensions taken from catalogues, operating voltage, the best arrangements and connection types, screening. In the electric circuit theory, such schemes are initial ones. They are taken in the ready-to-use form from other, more special electric engineering disciplines are subjected to theoretical analysis.

One should differentiate between the structural theoretical scheme and various types of real engineering schemes (e.g., wiring diagrams). Principal elements of the structural scheme are a power source, load (electric power receiver) and idealized structural elements, connecting them and represented by special symbols. Numerous parameters of real structural elements are omitted.

The "flow" scheme of the electric circuit theory reflects an electromagnetic process going in a functioning electric device and the circuit itself is a set of elements and their relations (connections), forming a current path. The latter has the following parameters: voltage, strength, power, amplitude, phase and frequency (for sinusoidal current). In addition, there exist various kinds of this process (and their respective modes of circuit function): direct and alternating, periodical and nonperiodic, steady-state and transient currents, etc. Current transformation is either the quantitative transformation of its parameters (for example, current strength and voltage) or the transformation of the pattern of its variation in time (say, of direct current into alternating current or vice versa). Resistance, inductance, capacitance, which are further idealizations of the corresponding structural elements of the electric circuit (the resistor, inductor, capacitor), and ideal current and voltage sources can be considered as "flow" scheme elements. This "semiotic constructor" makes it possible to represent any structural element of the structural scheme.

To each element of the "flow" scheme there corresponds a specific physical process whose detailed description is beyond the scope of the electric circuit theory which takes it into account, however. (For example, resistance represents irrecoverable losses of electric energy in the circuit, resulting from its transformation into other forms of energy-thermal, chemical, etc.) In the electric circuit theory, this process is expressed by a definite relationship of physical parameters of an element, say, voltage versus current strength or electric charge versus voltage, and the number of appropriate units of measurement (ohm, farad, hertz, etc.) Electric circuit elements form branches which are joined by means of ideal electric connections (i.e. connections free of resistance, inductance, capacitance) to form nodes and loops.

Similar in nanotechnology nanoinsulators and nanoconnectors for optical nanocircuits may be considered to be complex circuit elements, $C_{1}, C_{2}$ and $L$ (see Figure 2): "it is possible to characterize complex arrangements of (plasmonic and nonplasmonic) optical nanocircuit elements using the circuit theory" (Silveirinha, Alù, Li, \& Engheta, 2007: p. 64).

Distributed-parameter circuits ("A distributed parameter is a

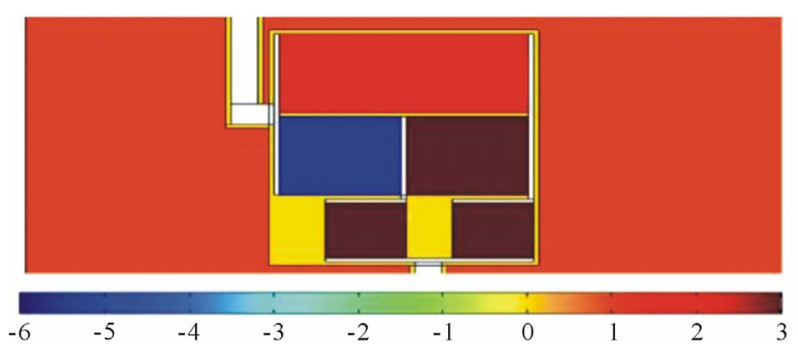

(a)

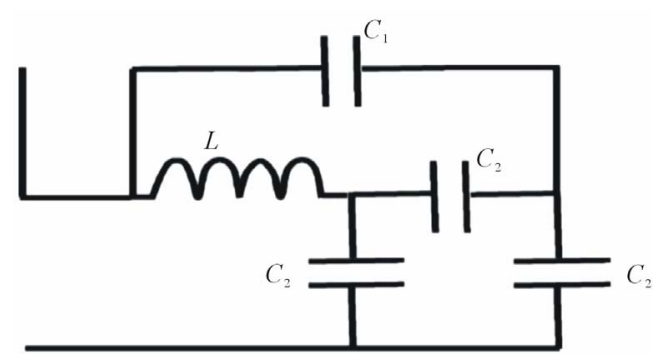

(b)

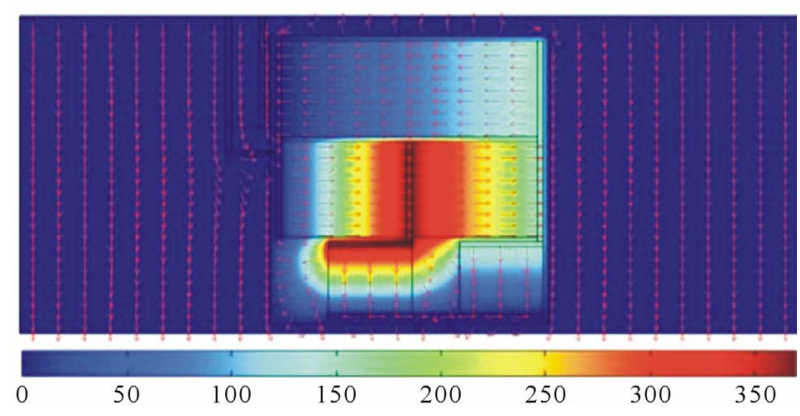

(c)

Figure 2.

(a) An optical nanocircuit formed by five nanomodules (four nanocapacitors and one nanoinductor), mimicking the function of the circuit shown in (b). Here a 2D configuration is considered. The value of the permittivity for each nanomodule is shown in the color scale in (a). The white region represents a material with a high permittivity (EVL). (c) Two-dimensional (2D) finite element method (FEM) "quasi-static" simulation of optical nanocircuit in (a). Here the color scheme shows the optical potential distributions, and the arrows shows the direction (not the amplitude) of displacement current in each nanomodule. We note how high the value of optical potential reaches in some of the nodes of this nanocircuit, due to the $\mathrm{LC}$ resonance (Silveirinha, Alù, Li, \& Engheta, 2007: p. 63). 
parameter which is spread throughout a structure and is not confined to a lumped element such as a coil of wire" (Wilson, 2007), e.g., homogeneous lines, are theoretically presented for engineering analysis as distributed-parameter circuits equivalent to them under given operating conditions (e.g., in a particular frequency band). The distributed-parameter circuit can be analyzed within the framework of the electric circuit theory and with the use of the electromagnetic field theory. Moreover, the flow scheme of substitution, derived within the framework of the electric circuit theory can be represented by different functional schemes (e.g., the potential diagram or two-ports). Similar in the nanotechnoscience can be described the geometry of two-nanotube transmission line and his RF circuit model (Burke, 2004: p. 3).

Functional schemes of the electric circuit theory are diagrams, graphical forms of the mathematical description of the electric circuit state, To each functional element of this diagram there corresponds a particular mathematical relationship, say, current strength versus voltage in some circuit section, or a particular mathematical operation (say, differentiation or integration). The arrangement and characteristics of functional elements correspond to the flow circuit scheme. Thus, in the circuit analysis, say, with the aid of the graph theory, circuit flow scheme elements (inductances, capacitances, resistances, etc.) are substituted, in accordance with definite rules, by a special ideal functional element - unistor, letting current to flow only in one direction. The resultant homogeneous theoretical scheme can be handled with the use of topological methods of circuit analysis (Starzyk \& Sliwa, 1984). Thus, the functional schematic circuit diagram corresponds to a particular equation set and, at the same time, it is equivalent to some flow scheme.

\section{Nanosystem as Electronic Nanocircuit-Models from the History of Science}

The nanomachines can be regarded as nanoelectrical switches in the nanocircuit. In nanotechnology define a nanomachne also as the nanocircuit. "Nanotechnological constructions are to reproduce traditional electronic components (switches, diodes, transistors, etc.) on a nanoscale. One main goal of this effort is to open up new dimensions of data processing, namely through the storage of large amounts of data in the smallest possible space... Because of the intermediary position of the nanoscale, it is also called "mesoworld"' (Schiemann, 2005).

In the nanocircuit structure we can find traditional electronic components ("molecular-scale electronic components") of the different levels realized on the base of the nanotechnology:

1) First of all, such electronic elements as an electronic switch (e.g. transistor), wires, inductors and capacitor or battery cell;

2) Second, electronic units (blocs) as antenna ("radiates transmitted power in narrow beam for maximum 'gain' and receives backscattered signal from targets") or modulator ("to 'trigger' the transmitter operation at precise and regularly recurring instants of time") (Barrett, 2000-2002: p. 23);

3) Third, complex nanosystems as a hole (e.g. nanocomputer).

\section{Nanoinductors, Nano-Capacitors, and Nano-Resistors}

In the Figure 3 you can see three basic circuit elements at optical frequencies - nanoinductors, nano-capacitors, and nanoresistors. "There is not that much difference between a battery

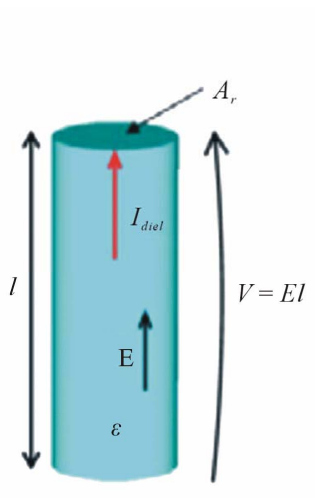

(a)

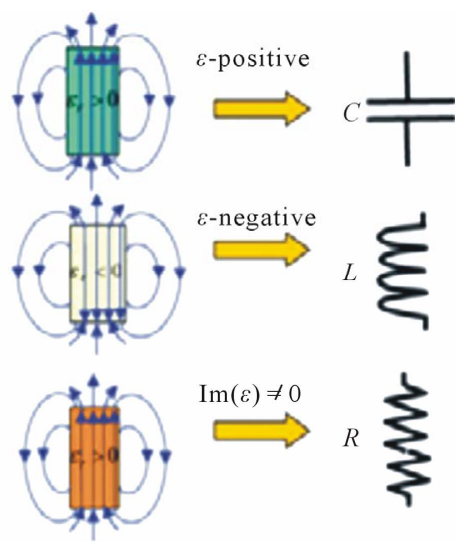

(b)
Figure 3.

(a) Geometry of a generic subwavelength nanocircuit element in the form of a nanowire with length $l$ and cross-section $T A$; (b) Equivalent circuit model for the nanowire depending on the electrical properties of the material (Silveirinha, 2007).

and a capacitor... Conventional Faraday capacitors store electric charge between parallel charged plates that are separated by an insulating dielectric material. Instead of flat parallel plates, capacitors that come in tubes use two metallic foils separated by an electrolyte-impregnated paper in a "sandwich" that is rolled up into the tube. For these devices, nanotube thin films can increase the surface area of the conducting foil due to the nanotubes' very small size, orderly alignment and high conductivity. "Nanotubes provide a huge surface area on which to store and release energy-that is what makes the difference..." (Johnson, 2005).

\section{Atomic-Scale Transistor and "Electronic Tube"}

In nanoscience, such wave processes are investigated at the level of the single electron, atom, or molecule, as well as of the cluster of atoms and molecules. And at the basis of this research, for example, of the the wave function, a new nanosystem can be constructed, which is in principle similar to radio equipment or to those of its elements, such as the atomic-scale transistor (see Figure 4), which "can be reversibly switched between a quantized conducting on-state and an insulating off-state by applying a control potential relative to a third, independent gate electrode" (Xie, 2007), or "electronic tube" as two-dimensional nanostructure. Electron transport in nanostructures on helium films (Leider \& Klier, 2008: p. 182). This is in principal similar with the three-electrode radio tube in the traditional electronic device. In engineering, schematic diagrams are more important than in science, since the peculiarity of engineering thought is operating with schemata and models. And these models adopt today from the history of science.

The atomic-scale transistor "can be reversibly switched between a quantized conducting on-state and an insulating offstate by applying a control potential relative to a third, independent gate electrode. For this purpose, an atomic-scale point contact is formed by electrochemical deposition of silver within a nanoscale gap between two gold electrodes, which subsequently can be dissolved and re-deposited, thus allowing open and close the gap". Here is the effect of this electrochemical cycling process and is discussed "the mechanisms of formation 


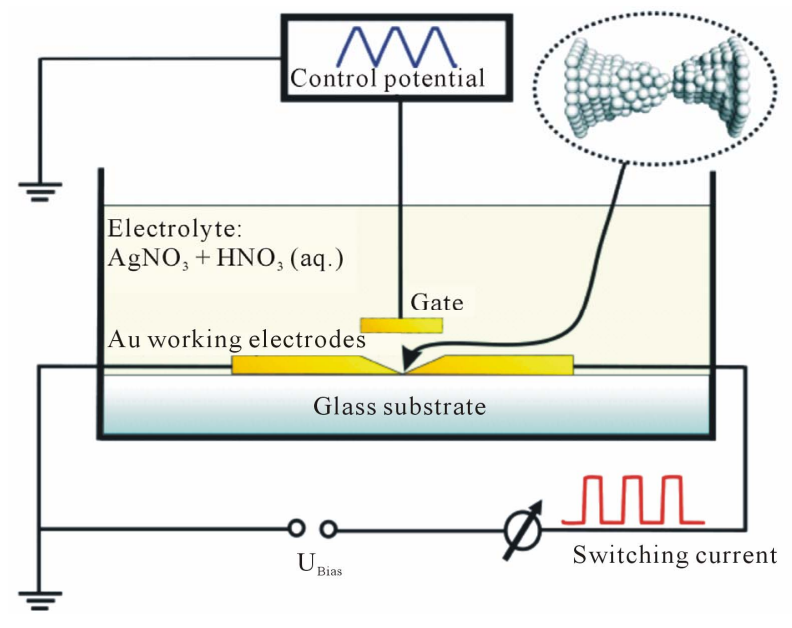

Figure 4.

Illustration of the experimental setup. "Silver quantum point contacts are electrochemically grown within a nanoscale gap between two electrodes deposited on a substrate. After repeated electrochemical deposition/dissolution processes, a bistable contact configuration is formed, and the reproducible switching of the contact between the two Au working electrodes is achieved by means of an independent gate electrode" (Xie, 2007: p. 115).

and operation of the atomic-scale quantum transistor" (Xie, 2007: p. 115).

In recent study of the nanotechnoscience is constructed "a molecular logic gate in a microfluidic system based on fluorescent chemosensors by detecting the changes in intensity as a response to various inputs (pH, metal ions)" (Berger, 2007) (see Figure 5). In principle mode of functioning of this electronic switch not differ from the coherer-an electrical component formerly used to detect radio waves, consisting of a tube containing loosely packed metal particles (filing in coherer of Branly (see Figure 6) by Popov's receiver or nickel powder (by Marconi). The waves caused the particles to cohere, thereby changing the current through the circuit (see Gorokhov, 2006: pp. 21-22).

\section{Miniaturized Antenna on the Micro- and Nanometer Scale}

We can speak about for instance nanoantenna sensors in the visible and infrared regime: "In order to detect electromagnetic radiation, one needs two basic elements: 1) a physical structure that efficiently couples to the radiation - the antenna; and 2) a rectifying element that converts the high-frequency $\mathrm{AC}$ signal to a low-frequency signal that can be detected by electronic means. Antenna structures and rectifying diodes have long been studied and applied for radio waves, television signals, cell phones, and so on. Recent work has shown that miniaturized antennas on the micro- and nanometer scale can be tuned to infrared and visible radiation, and that these nanoantenna structures can be integrated with metal-oxide-metal (MOM) rectifying diodes. The sensor consists of a MOM diode integrated together with a dipole antenna" (Bernstein, 2006: pp. 133-138).

Analogy between an early Hertzian antenna to operate at microwave frequencies and the nanodimer antenna see in Figure 7. "The pioneering work of Hertz at the end of the nineteenth century is at the foundation of the modern antenna science and engineering, and therefore of an important part of current wire-

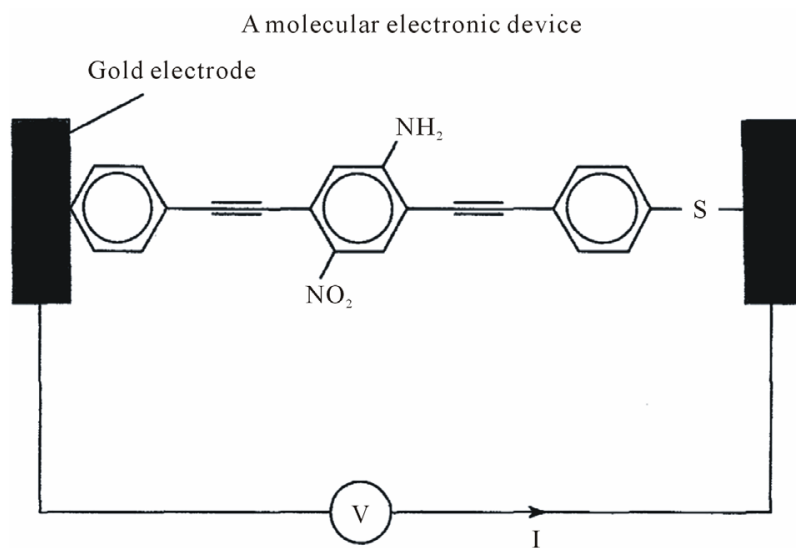

Figure 5.

"Illustration of an electronic switch made of a conducting molecule bonded at each end to gold electrodes. Initially it is nonconducting; however, when the voltage is sufficient to add an electron from the gold electrode to the molecule, it becomes conducting. A further increase makes it nonconducting again with addition of a second electron" (Pool \& Owens, 2003: p. 351).

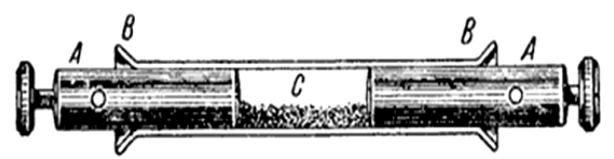

Figure 6.

Coherer of branly (Gorokhov, 2006: p. 48).

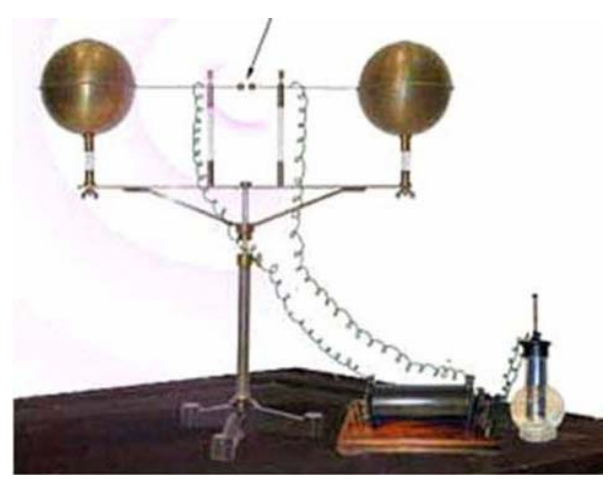

(a)

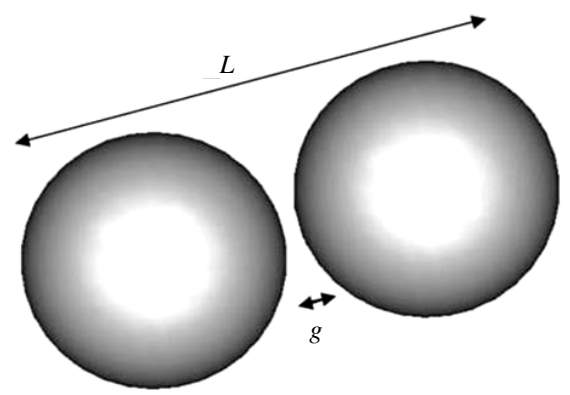

(b)

\section{Figure 7.}

Analogy between two dimer antennas: (a) An early Hertzian antenna to operate at microwave frequencies; (b) The plasmonic nanodimer antenna in the form of two closely spaced spherical nanoparticles (Alù, 2008: 195111-1). 
less technology. His intuition of driving oscillating charges distributed over two closely spaced spherical capacitors... has proven successful for generating the first class of working radiators, and it has paved the way to myriads of wireless applications in the current technology... Currently, the theory and practice of RF antenna design is well established, and the old geometry of Hertz's first antennas... would definitely look outdated, compared with the myriad of different antenna designs currently available for numerous different purposes and applications... However, for different reasons the optical nanoantenna science is still in its early stage, and the recent experiments on optical nanoantennas may be well compared with the first attempts performed by Hertz... In this context, we have recently proposed a general theory that may bring and utilize the concepts of input impedance, radiation resistance, antenna loading, and matching of optical nanoantennas in order to translate the well-known and established concepts of RF antenna design into the visible regime" (Alù, 2008: 195111-1). This is right for nanocircuits at all.

\section{Micrometer-Scale Silicon Electro-Optic Modulator}

In radio electronics and radiolocation, modulation is the process of varying one or more properties of a high frequency periodic waveform to receive a modulating signal with help of modulator. "Because of the high rate of switching (many hundreds of pulses per second) and the very short time intervals being used (a few microseconds at the most for the pulse duration) the transmitter operation cannot be controlled by normal switches or relays. The circuit which does this switching, and also supplies the input power required by the oscillator, is the modulator. It is an electronic circuit which is 'triggered' by the output from the master timing unit and which produces a d.c. pulse whose duration is determined by the circuitry of the modulator. This d.c. pulse of controlled pulse duration, recurring at the precise instants of time determined by the master timing unit, is used to switch the oscillator on and off (Barrett, 2000-2002: p. 16). The same nanoblock as modulator we can see in the nanotechnology. "Much of our electronics could soon be replaced by photonics, in which beams of light flitting through microscopic channels on a silicon chip replace electrons in wires. Photonic chips would carry more data, use less power and work smoothly with fiber-optic communications systems. The trick is to get electronics and photonics to talk to each other... Now Cornell University researchers have taken a major step forward in bridging this communication gap by developing a silicon device that allows an electrical signal to modulate a beam of light on a micrometer scale... Their modulator uses a ring resonator - a circular waveguide coupled to a straight waveguide carrying the beam of light to be modulated. Light traveling along the straight waveguide loops many times around the circle before proceeding... The ring is surrounded by an outer ring of negatively doped silicon, and the region inside the ring is positively doped, making the waveguide itself the intrinsic region of a positive-intrinsic-negative (PIN) diode. When a voltage is applied across the junction, electrons and holes are injected into the waveguide, changing its refractive index and its resonant frequency so that it no longer passes light at the same wavelength. As a result, turning the voltage on switches the light beam off... The PIN structure has been used previously to modulate light in silicon using straight waveguides. But because the change in refractive index that can be caused in silicon is quite small, a very long straight waveguide is needed. Since light travels many times around the ring resonator, the small change has a large effect, making it possible to build a very small device. Tests using a pulse-modulated electrical signal produced an output with a very similar waveform to the input at up to 1.5 gigabits per second" (Steele, 2005).

\section{Nanotechnology-Complex Electronic Circuitry with Multiple Junctions and Interconnects}

An important area for development within molecular manufacturing is systems design of the extremely complex molecular systems. "Although the design issues are likely to be largely separable at a subsystems level, the amount of computation required for design and validation is likely to be quite substantial. Performing checks on engineering constraints, such as defect tolerance, physical integrity, and chemical stability, will be required as well" (Arnall, 2003: p. 37). "Because the switches are so tiny, they operate in the realm of quantum physics, which opens the possibility of using the switch to make a multi-bit memory device... The researchers also used the switches to form the basic binary logic gates required to make computer processor chips. They made an AND gate using two switches formed from a single silver sulfide wire and two platinum wires combined with a resistor that restricts electric currents to specific voltages. An AND gate produces a 1 only if both inputs are 1. They made an OR gate using two switches formed from two silver sulfide wires and a single platinum wire combined with a resistor. An OR gate produces a 0 only if both inputs are 0 . They made a NOT gate using one switch combined with two resistors and a capacitor, which briefly stores electric charge. A NOT gate turns an input of 1 into 0 and vice versa" (Smalley, 2005).

\section{Analysis and Synthesis of the Nanocircuits from the Point of View of the Electrical Circuits-Historical Transfer of the Methodology for the Research of the New Types of the Technical Systems}

Following the paradigm of the electric circuit theory nanocircuits may be considered in different frequency regimes as complex circuits consists of the three basic elements of any linear circuit, R, L, and C. For example, pass-band optical nanofilter can be described as parallel RLC resonance (see Figure 8) and stop-band optical nanofilter als series LC resonance. fabricating nanofilters in optical lumped nanocircuit devices... The importance of transplanting the classical circuit concepts into optical frequencies is based on the possibility of squeezing circuit functionalities (e.g., filtering, waveguiding, multiplexing...) in subwave length regions of space, and on correspondingly increasing the operating frequency with several orders of magnitude. Moreover, nowadays the interest in combining optical guiding devices, as optical interconnects, with micro- and nanoelectronic circuits is high..., since it is "Following the nanocircuit theory, we show how it is possible to design such complex frequency responses by simple rules, similar to RF circuit design, and we compare the frequency response of these optical nanofilters with classic filters in RF circuits. These results may provide a theoretical foundation for not still possible to perform all the classic circuit operations in the optical domain. Introducing new paradigms and feasible methods to bring more circuit functionalities into the optical domain would rep- 

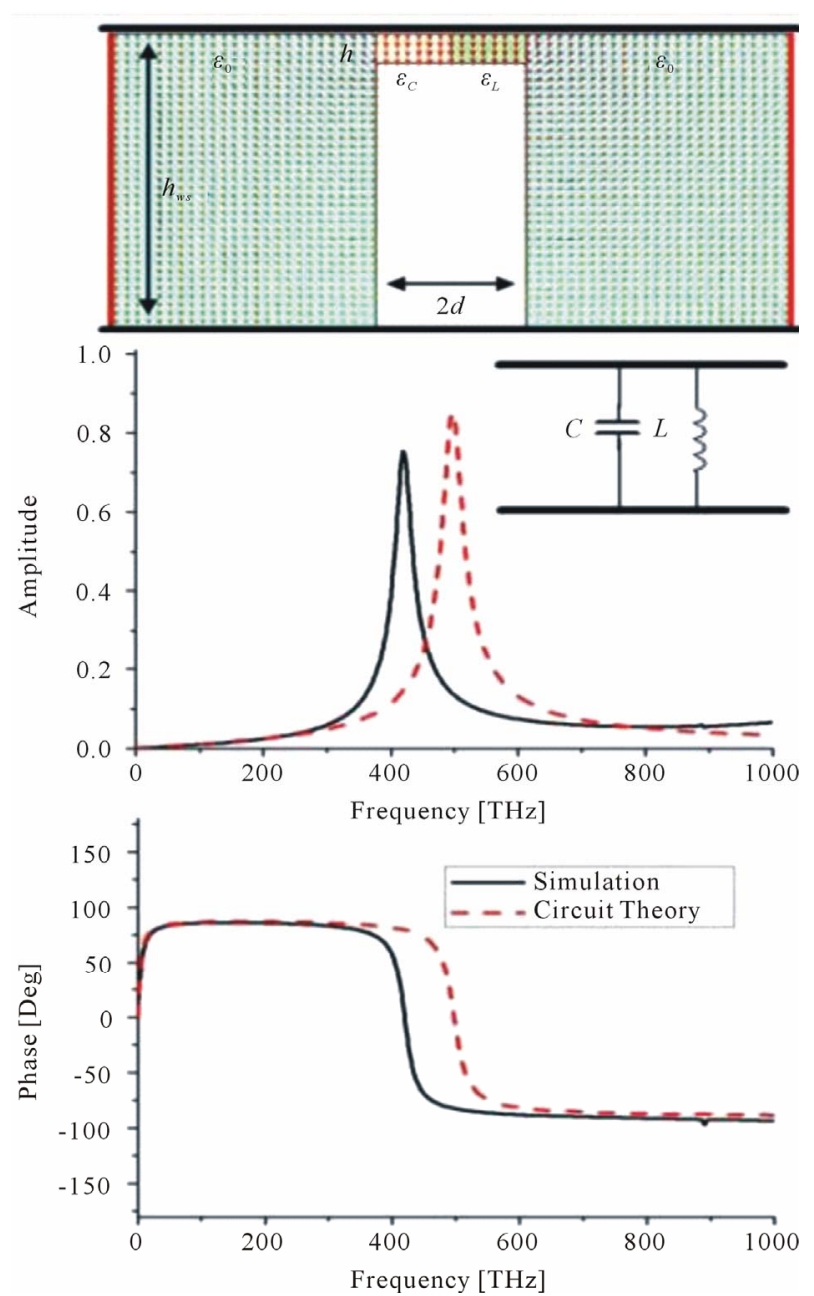

Figure 8.

Transfer function (amplitude and phase) and electric field distribution at the resonance for an optical pass-band nanofilter formed by two nanorods juxtaposed in parallel in a waveguide, one made of silicon and the other made of silver (Alù, Youngy, \& Engheta, 2008: 144107-4).

resent an important advance in nanoelectronics technology... we have introduced and discussed the fundamental concepts for developing a novel paradigm for optical nanocircuits, with the aim to extend classic circuit concepts, commonly available at $\mathrm{RF}$ and lower frequencies, to higher frequencies and in particular to the optical domain. Specifically, we have discussed... how a proper combination of plasmonic and non-plasmonic nanoparticles may constitute a complex nanocircuit at infrared and optical frequencies, for which the conventional lumped circuit elements are not available in a conventional way. After introducing the nanocircuit concepts for isolated nanocircuit elements..., and after having applied them to model infinite stacks of nanoelements to design nanotransmission lines and nanomaterials..., we have been interested in analyzing in details how the connections and interactions among the individual nanoelements may be modeled and designed in a complex optical nanocircuit board with functionalities corresponding to those of a classic microwave circuit" (Alù, Youngy, \& Engheta, 2008: 144107-1).

In principle, the both procedures analysis and synthesis are similar since the synthesis of a new technical system involves the analysis of the existing similar devices.

The engineering theory function is by "shuttle" iteration. First, an engineering problem consisting in construction of some technical system is formulated. Then it is represented as an ideal structural scheme which is then transformed into a natural process scheme showing technical system function. To analyze and mathematically model this process, a functional scheme representing particular mathematical relationships is constructed. The engineering problem is thereby reformulated into a scientific problem, and then into deductively solved mathematical problems. This upward way is termed the ana1ysis of schemes.

The reverse way - the synthesis of schemes - makes it possible to use the available structural elements, more specifically the corresponding abstract objects, to synthesize a new technical systems (more specifically, its ideal model, theoretical scheme) in accordance with definite rules of deductive transformation, calculate basic parameters of the object and simulate its function. The solution obtained at the ideal model level is gradually transformed to the engineering level where such engineering parameters as overall dimensions and weight of parts, types of connections, connection and part screenings from side electromagnetic effects, the best structural arrangements, etc., considered to be secondary parameters from the ideal model viewpoint, are taken into account and additional theory-correcting computations are performed. Thus, the lower level of engineering-theory abstract objects (structural schemes) directly involves empirical (structural \& technical and manufacturing) knowledge, and is intended for utilization in engineering. It is this last fact that largely determines the specific feature of design-oriented engineering theory: to its abstract objects there must correspond a class of hypothetical technical systems which have not been created yet. Therefore, both analysis and synthesis of theoretical schemes of technical systems are important in engineering theory (see Figure 1).

In the analysis of an electric circuit in the electric circuit theory, the initial scheme is a structural diagram of an electric device. In conformity with the problem being solved, it is substituted by an equivalent flow scheme valid for the functional mode of the device, the substitution being done in accordance with special rules. Further transformations of the latter scheme are aimed at obtaining simpler schemes which will be more suitable for computations. With this aim in view, special theorems are proved, definite scheme transformation rules formulated and standard design methods described. The synthesis of schemes consists in finding electric circuit elements which can ensure the required functional mode meeting the conditions specified in the form of a certain mathematical relationship. To simplify synthesis, use is made of standard schemes, tables of standard circuits and corresponding mathematical relationships. In engineering practice, pure synthesis is extremely rare; certain parameters of a technical system and its elements are generally specified as early as in the problem statement and synthesis is often reduced to mere updating of an earlier device. Moreover, engineering practice always uses traditional empirical structural schemes, usually ready-to-use ones. Therefore, synthesis is reduced to analysis and what is to be determined is a few parameters of the newly designed circuit. At this stage the engineer often resorts to iteration methods, based on successive approximation; he approaches to the solution step by step, returning to the initial problem more than once. In mature engineering practice associated with mass and series production, 
technical systems are constructed of standard elements. Therefore, in theory, synthesis also involves the combination of standard idealized elements in accordance with standard rules of theoretical scheme transformation. Analysis is also reduced to the same procedure.

It is possible to extend the classic circuit concepts, commonly available at microwave and lower frequencies, to higher frequencies and in particular to the optical domain (Figure 9). "We have developed accurate circuit models at optical wavelengths to characterize the equivalent impedance of the envisioned nanocapacitors and nanoinductors. It has also been shown that the induced displacement current may leak out of the subwavelength nanocircuit elements, causing strong coupling between the nanoelements and the neighboring region. To circumvent this problem, we have introduced the concept of optical nanoinsulators for the displacement current... We have confirmed, both analytically and numerically, that nanocircuit elements... may be accurately characterized using standard circuit theory concepts at optical frequencies, and in particular they may indeed be characterized by an equivalent impedance for nanocircuit elements. We have further explained how to apply the proposed circuit concepts in a scenario with realistic optical voltage sources. We have also studied how to ensure a good connection between the envisioned lumped nanoelements... This has led us to consider unit nanomodules for lumped nanocircuit elements, which may be regarded as building blocks for more complex nanocircuits at optical wavelengths" (Silveirinha, Alù, Li, \& Engheta, 2007: p. 64).

Analytical quasi-static circuit models ("modeled theoretically") for the coupling among small nanoparticles excited by an optical electric field in the framework of the optical lumped nanocircuit theory in Figure 10 are of importance in the understanding of complex optical nanocircuits at infrared and optical frequencies.
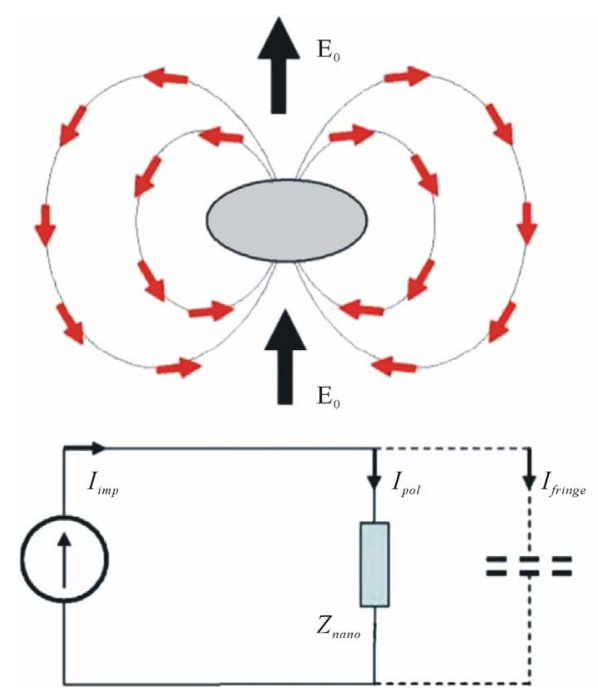

Figure 9.

(Color online) A nanoparticle illuminated by a uniform optical electric field $\mathrm{E}_{0}$ (black arrows) may be viewed in terms of the circuit analogy presented... as a lumped impedance nano $Z$ excited by the impressed current generator imp I and loaded with the fringe capacitance associated with its fringe dipolar fields (red arrows) (Alù, Salandrino, \& Engheta, 2007).

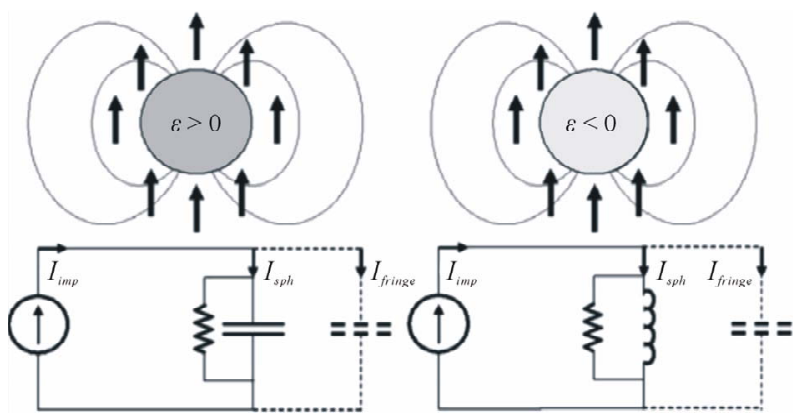

Figure 10.

A basic nanocircuit in the optical regime, using the interaction of an optical wave with an individual nanosphere. (left column) A nonplasmonic sphere with $\varepsilon>0$, which provides a nano-capacitor and a nano-resistor; (right column) A plasmonic sphere with $\varepsilon<0$, which gives a nano-inductor and a nanoresistor. Solid arrows show the incident electric field, and the thinner field lines represent the fringe dipolar field from the nanosphere (Engheta, Salandrino, \& Aiu, 2004: p. 12).

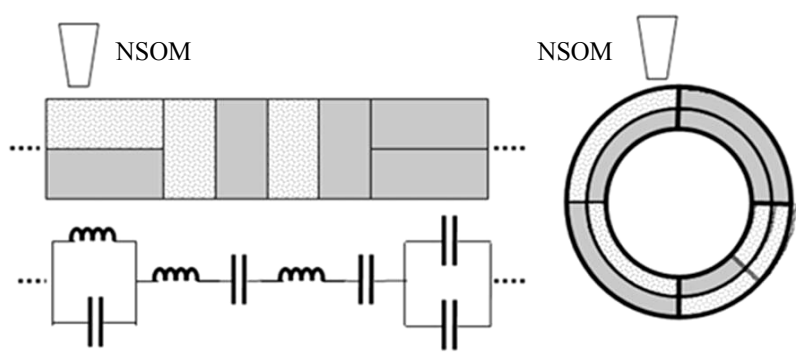

Figure 11.

Nanocircuit synthesis. (Top left) Conceptual nanocircuit formed by rectangular blocks of plasmonic and non-plasmonic segments; (bottom left) Its equivalent circuit; (right) A closed "nano-loop" (Engheta, Salandrino, \& Aiu, 2004: p. 13).

Synthesizing nanocircuit elements in the optical domain using plasmonic and non-plasmonic nanoparticles from three basic circuit elements, i.e., nanoinductors, nano-capacitors, and nano-resistors see for example in Figure 11.

"All these concepts are important steps towards the possibility of synthesizing a complex optical nanocircuit board with the functionalities analogous to a classic microwave circuit (e.g., filtering, waveguiding, multiplexing...)". Such approach "would allow one to quantitatively design and synthesize desired nanocircuits (such as nanofilters, nanotransmission line, parallel and series combination of nanoelements, etc.) at optical frequencies using properly designed collections of nanoparticles acting as "lumped" nanocircuit elements. This concept may open doors to design of more complex nanocircuits and nanosystems in the optical domains" (Alù, Youngy, \& Engheta, 2008).

This methodology is typical for the engineering sciences at all and was developed already in the theory of mechanisms in the end of the 19th century. For example Fr. Reuleaux defines in his "Kinematics of Machinery. Outlines of a Theory of Machines" (Reuleaux, 1875) kinematic analysis and synthesis as follows: Kinematic analysis consisted in decomposing the existing machines into their component mechanisms, chains, links and pairs of elements, i.e., in determining the kinematic composition of the machine involved. The final result of that analysis was the choice of kinematic pairs, links, chains and mechanisms to be used to assemble a machine for carrying out the required motions. Reuleaux differentiated between direct and 
indirect synthesis. The former concerned the compositions of mechanisms which could effect particular changes of the body worked. This was possible when the mechanism was reduced to a kinematic pair. In that situation, the solution was the choice of a proper design for the elements of that pair. According to Reuleaux, the main method of theoretical synthesis of new mechanisms was indirect synthesis, i.e., the preliminary solution of all problems of a particular type, among which the method sought could be found. Such synthesis was possible because the number of realizable mechanisms was limited. First, all possible simple chains were investigated, which could be used to obtain a number of mechanisms by changing the ratio of various links to that chain, transforming some links of that chain into a fixed member, replacing some mechanism pair by another one, etc.

The operation of nanotheory is realized also as in the engineering theory by the iteration method. At first a special engineering problem is formulated. Then it is represented in the form of the structural scheme of the nanosystem which is transformed into the idea about the natural process reflecting its performance. To calculate and mathematically model this process a functional scheme is constructed. Consequently, the engineering problem is reformulated into a scientific one and then into a mathematical problem solved by the deductive method. This path from the bottom to the top represents the analysis of schemes (the bottom up approach). For instance, this can be the investigation of "the possibility of connecting nanoparticles in series and in parallel configurations, acting as nanocircuit elements" (Salandrino, 2007). The way in the opposite directionthe synthesis of schemes (the top down approach) - makes it possible to synthesize the ideal model of a new nanosystem from idealized structural elements according to the appropriate rules of deductive transformation, to calculate basic parameters of the nanosystem and simulate its function. Nanocircuit synthesis can be, for example, a synthesizing nanocircuit elements in the optical domain using plasmonic and non-plasmonic nanoparticles (Engheta, Salandrino, \& Aiu, 2004).

\section{Conclusion}

Thus, the engineering theory function consists in solving particular engineering problems with the aid of theory-evolved procedures, type analyses which are suitable in various, more special (scientific and engineering) studies and engineering practice. The creation of new procedures of this kind, the elaboration of rules and proofs of theorems concerning the adequacy of equivalent transformations and allowable approximations, the construction of new standard theoretical schemes pertains to the engineering theory advance on the frontiers of the theoretical research in engineering sciences, and its findings, are stated in primary publications (first of all, in articles) whereas textbooks and monographs provide examples of the engineering theory function, theoretically classify and systematize proven methods of engineering problem solution, demonstrate their compatibility with the general system of theoretical knowledge of the engineering discipline involved. In the natural scientific theory primary importance are flow schemes, but not structural schemes. Both the mathematical apparatus and experiments are for natural scientist just a means of prediction and explanation of the natural processes. For example, Hertz in principle worked as an engineer, when designing new experimental equipment. But he did not mean to find some technical application for his experimental devices. One of the major problems of the well-developed engineering theory function in "copying" of type structural schemes for various engineering requirements and conditions. Then the solution of any engineering problems, the construction of any new systems will be theoretical supported. This is the essence of the constructive function of engineering theory (theory in engineering science), its lead of engineering praxis. His solution result is cast into practical-methodical recommendations (for designer, inventor, production engineer, etc.). To its abstract objects there must correspond a class of hypothetical technical systems which have not been crated yet. Therefore, in the engineering theory is important not only analysis, but first of all synthesis of theoretical schemes of technical systems. So nanotechnology is at the same time a field of scientific knowledge and a sphere of engineering activity, in other words-NanoTechnoSciencesimilar with Systems Engineering as the analysis and design of complex man/machine systems but now as large-scale microand nanosystems. That is why is very important to investigate the historical sources of the nanotechnological methods in the history of science and technology.

The engineering theory function is aimed at approximation of the theoretical image of an technical system, its equivalent transformation into some new, simpler scheme which will be more suitable for computations, at the reduction of complex cases to simpler and standard ones for which a ready-to-use solution exists, Therefore, the major attention of the engineering theorist is directed at evolving standard solutions of engineering problems, standard design-simplifying methods. It also largely determines the nature of engineering theory supporting the validity of such equivalent transformations and approximations. No matter whether the analysis, synthesis of schemes or mere engineering computations are done, the following general "algorithm" of engineering theory function can be formulated (see Figure 12).

1) In the starting point of the process of the theoretical solution of a new engineering problem, the initial conditions of this problem, engineering requirements and limitations and possible analogies with previously solved problems are formulated in terms of structural \& technical and manufacturing knowledge. This procedure can be termed the engineering problem conceptualization.

2) The empirical description shall be theoretically formulated in concepts and notions which are standard for the engineering theory involved. This procedure can be termed the identification of the engineering problem with a scientific problem, i.e. the setting-up of a correspondence between the technical system under design and investigation and a particular theoretical scheme of the engineering theory involved. The result is a structural scheme constructed of idealized elements taken from a standard elements catalogue.

3) The so constructed structural scheme is transformed into a simpler type scheme by the first-order approximation. The transformation is accompanied by singling out technical system parameters which are the most important in the problem involved. Equivalent transformations (the formation of substitution schemes) are used to form flow schemes for various modes of technical system function, specified in the problem statement. If a complex flow scheme cannot be approximated, in one or several steps, to the simplest type diagram for which there exists a standard theory - evolved solution (if even these manipulations are not required, the solution is found directly from table 


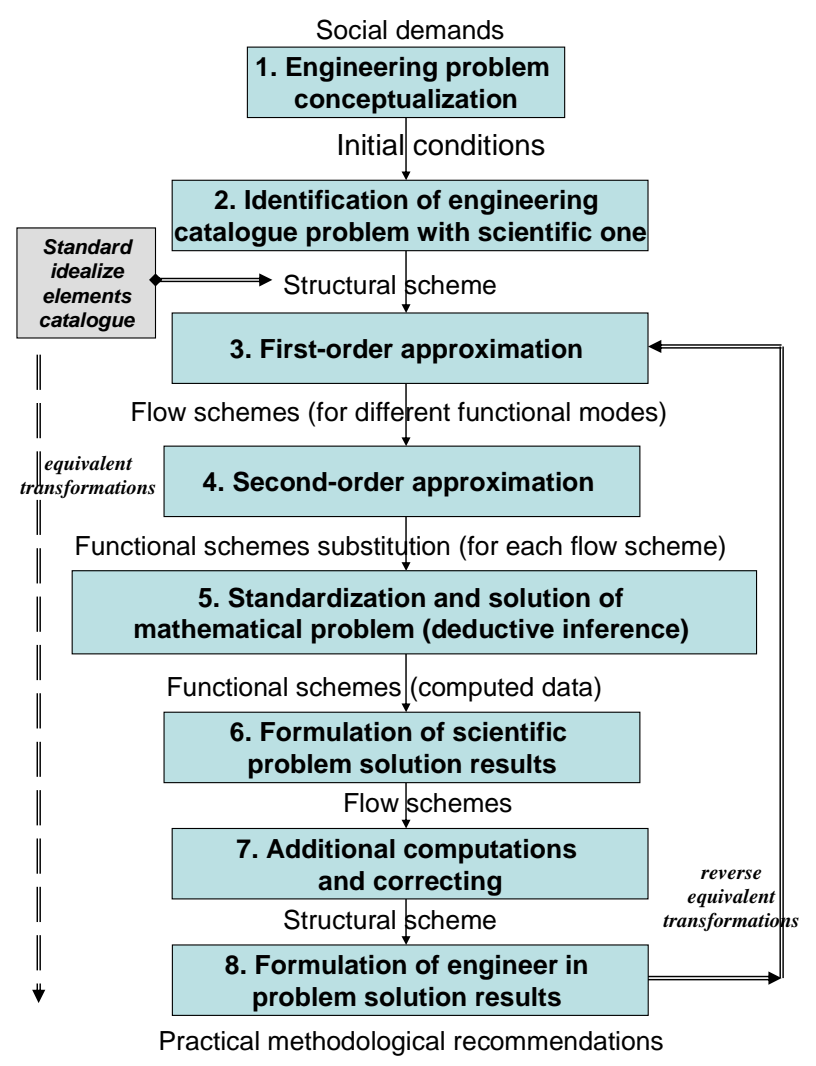

Figure 12.

General algorithm of engineering theory function.

formulas), it is substituted by an equivalent functional scheme in accordance with definite rules of correspondence.

4) A functional scheme constructed with the aid of the second - order approximation is used to formulate an equation set to be solved by special mathematical methods (e.g., by matrix ones). These equations are obtained on the basis of physical (Ohm's, Kirchhoff's and other) laws setting up, for example, a relationship between circuit current parameters and circuit element parameters. Their concrete numerical values known from the problem statement make it possible to determine unknown current and circuit element parameters through solving the equations.

5) The functional scheme is used to solve the mathematical problem using a standard computational procedure and standard problem solution methods based on previously proved theorems. To this end, the functional scheme is reduced to a standard one in accordance with definite rules of substitution. Thus, in the electric circuit theory, mixed connections are transformed into simpler, series and parallel ones, multiloop circuits are turned into single-loop ones, etc. In the electric circuit theory, such simplifying transformations are based on specially proved equivalence of some type schemes (e.g., of a "delta" and "star" and vice versa) and relevant theorems (say, the equivalent current and voltage source theorem) which give more computationally suitable schemes. This makes it possible to substitute certain circuit sections by other, equivalent and scheme-simplifying ones. The problem solution result obtained, by mathematical methods is translated to the flow scheme level by reverse equivalent transformation. Scientific problem solution results are formulated. Several flow schemes (for various functional modes) are then synthesized into an engineer object structural model.

7) Then the solution is adapted to a specific case and partly modified, i.e. additional computations are done and structural and engineering amendments introduced. It is necessitated by the fact that both the analysis and synthesis of schemes are invariably based on a compromise, trade - off between the complexity and accuracy of computations, on approximate methods and standard artificial techniques. The findings of theoretical computations must be corrected to take account of various engineering, social, economical, ecological and other requirements. It may call for the incorporation of new elements satisfying these requirements into theoretical schemes; these elements may be considered as connotations (additional, accompanying attributes) of these schemes. Framing a system of connotations which are incorporated into engineering-theory theoretical schemes as special elements may make it necessary to multiply return to previous stages (the iteration procedure) in order to construct new flow and structural schemes (corrected for these connotations), perform new approximations, equivalent transformations and computations. One of the major problems of well-developed engineering theory function is "copying" of type structural schemes for various engineering requirements and conditions. Then the solution of any engineering problems, the construction of any new engineering systems of a given type will be theoretically supported. This is the essence of the constructive function of engineering theory, its lead of engineering practice. Otherwise its function will amount only to solving routine engineering problems.

8) The final procedure of engineering theory function is that the solution result is cast into practical methodological recommendations (for the designer, inventor, etc.).

The constructive application of nanoscience as technoscience is expressed in its guidance of development in engineering practice. In nanotechnoscience, therefore, a prediction of the flow of natural processes on the nanolevel is just as important as the replication of the structural diagram of a new nanosystem (for example, a spintronic component, such as the "spin valve"). The superconductivity re-entrant phenomenon opens genuine prospects for building a very rapidly operating device, the "superconducting spin valve" for superconducting spintronics. Graphene electronics could even manipulate electrons as quantum-mechanical waves (similar to light waves made up of photons) rather than as particles.

It is very important to differentiate real fabricated "large scale MEMS" or "large-scale carbon nanotube devices" as three dimensional nanostructures from equivalent circuit modelled their components. Figures 13(a) and (b) show "scanning ion microscope (SIM) image of inductor (L), resistor (R) and capacitor $(\mathrm{C})$ in a parallel circuit structures with free space nanowiring" (Bhushan, 2004: p. 187).

The "electrical engineering" schematic diagrams reflect physical processes which take place within the elements and units of radio engineering devices. Such diagrams deal with the calculation of parameters and the mapping of electric currents in standard electrical elements such as resistors, capacitors, and inductors. Of course, these devices can be called electrical circuit only with reservations. Use is made of electronics theory to describe the physical processes in the new radio engineering elements such as, for example, electron tubes or semiconductor devices. But to calculate of the parameters of these devices in which they are included use is, as a rule, made of traditional equivalent circuit (resistors, capacitors and inductors). As the 
physical processes in elements of radiolocation devices (klystrons, magnetrons, cathode ray tubes, antennas, etc.) operating in new radio engineering regimes are different, it was necessary to modify the former methods of their calculation and representation or to develop new ones, as well as to develop new mathematical resources. The process was also stimulated by the need to investigate and develop methods of internal noise suppression in elements of radiolocation equipment (for example, the schrot effect in electron tubes). Similar is in the nanotechnology.

It is well-known that, in nanotechnoscience, constructs from various scientific theories-classical and quantum physics, classical and quantum chemistry, structural biology, etc.- are used, whereas, in nanosystems, different physical, chemical and biological processes take place. One can, however, also construct a circuit on the basis of definite nanosructures, such as, e.g., a super-heterodyne radio receiver on the nanolevel (Figure 14).

One of the important methods in the engineering sciences and also nanotechnscience is an approximation. The implementation of engineering theory involves a sequence of socalled approximations. For example, in electronics, the two-port theory is used to analyze complex circuits, the parameters of which are difficult to determine, owing to the awkwardness of the computations. Approximation is the substitution of some mathematical functions or designs by other, very similar, simpler functions or designs, which are equivalent in the desired aspect and for which known solutions exist, or can easily be obtained. In engineering sciences, this is a method for solving engineering problems on the basis of theoretical models and with the aid of a series of equivalent substitutions and transformations. The method of approximation is essentially a compromise between the accuracy and the complexity of designs. Accurate approximation usually involves complex mathematical relationships and computations. An oversimplified equivalent scheme of a technical system affects the accuracy of computations. The approximating expression or scheme must ex- press the nature of the function or scheme under approximation as accurately as possible, and be as simple as possible, in order to simplify the mathematical solutions of engineering problems. Any approximation calls for a special substantiation of solution adequacy, one type of approximation being preferable for one functional mode and other types being preferable for othermodes).

The two-port concept is introduced to facilitate the transition to mathematical relationships, making it possible to apply Kirchhoff's laws, which describe the natural process of current flow in the two-port circuit, and the corresponding equations in the matrix form. The coefficients of these equations are called two-port parameters, because they are determined solely by the two-port's properties. By solving these equations with the aid of the matrix theory, one can determine the structural parameters of two-ports sought-input resistance, input and output

\section{L, C, R Circuit Structure}

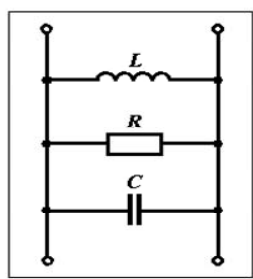

Growth time: $20 \mathrm{~min}$

(a)

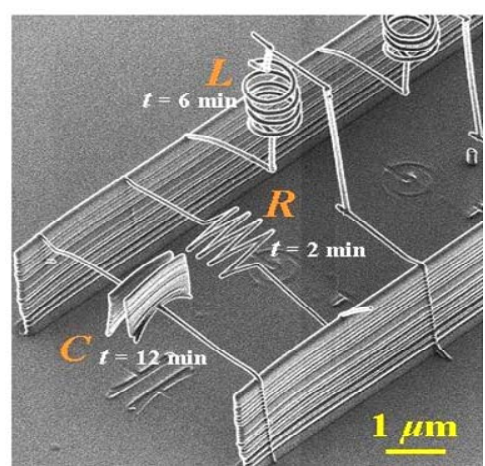

(b)
Figure 13.

Scanning ion microscope (SIM) micrograph of inductor (L), resistor (R) and capacitor (C) structures: (a) equivalent circuit modelled (b) three dimensional nanostructure (Bhushan, 2004: p. 186).

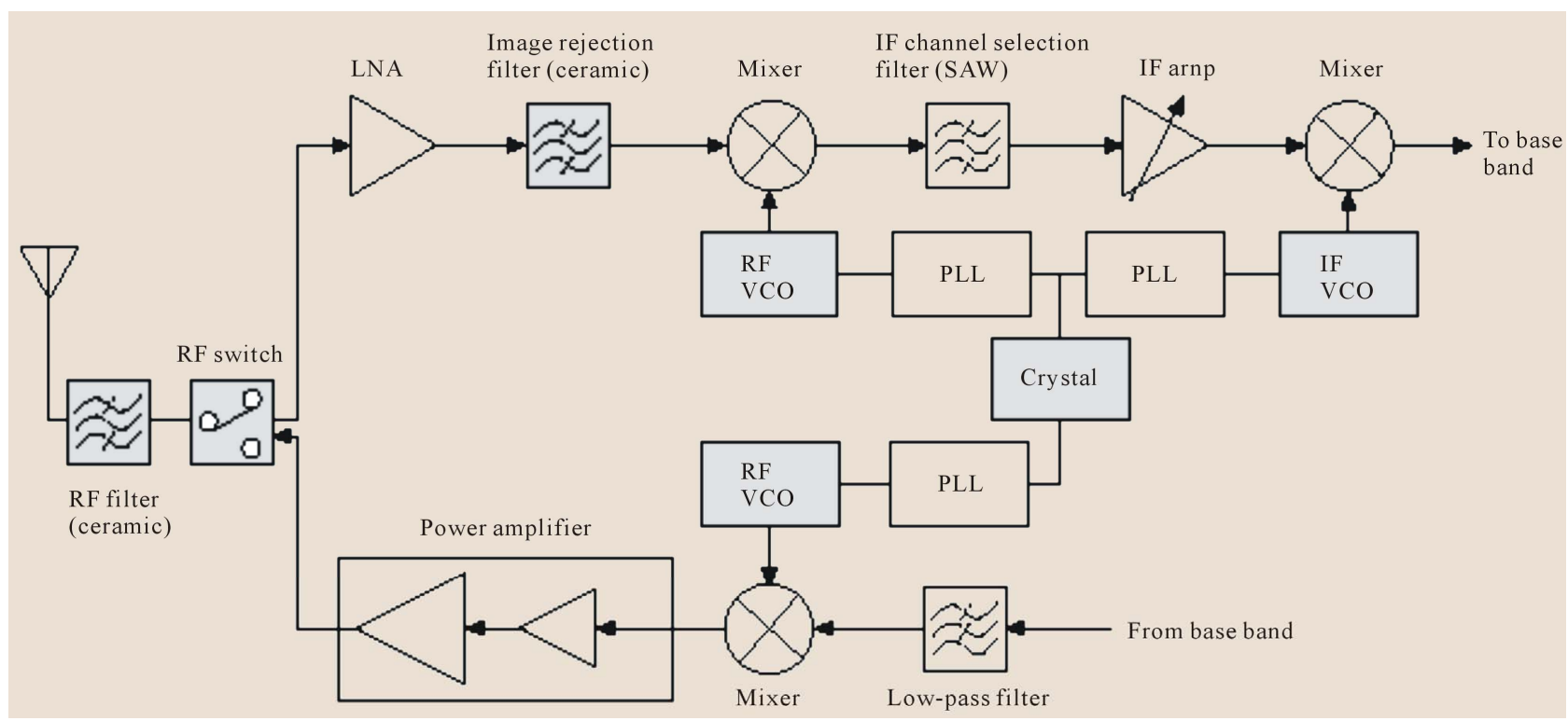

Figure 14.

Schematic of a super-heterodyne radio architecture (VCO = voltage-controlled oscillators, radio frequency (RF) and intermediate frequency (IF), $\mathrm{SAM}=$ self-assembled monolayer, $\mathrm{PLL}=$ phase-locked loop, LNA = low-noise amplifier) (Bhushan, 2004: p. 240). 
power, insertion loss, etc. A number of theorems (the reversibility theorem, equivalent oscillator theorem, etc.) are proved in two-port theory. Its use makes it possible not only to simplify the computations, but also to synthesize new models by deductive equivalent transformation of two-ports. Such a transformation gives the most economical and effective engineering solutions. It indicates natural restrictions on these transformations, the main types of two-ports and the types of their connections. It should be noted that, in analyzing complex circuits, these are preliminarily transformed into a combination of simpler twoports, the parameters of which are taken from special tables. Matrices for each of them are then used to carry out mathematical operations (addition, multiplication, etc.), depending on their connection type.

Several types of mathematical methods correspond to the same engineering theory. This is due to the fact that ideal objects are investigated at different levels. We have just considered the two-port theory and its mathematical apparatus. However, electric circuit analysis also involves the concept of a one-port making up larger structural "building-blocks", or units. (The one-port is a two-pole circuit section to which a difference of potentials is applied and which carries current.) Any amplifier, oscillator, filter, etc. can be considered to be a sum of capacitors, inductors, resistors, current and voltage sources. The latter are also idealizations, i.e., circuit theory deals with a comparatively small number of ideal elements and their combinations, representing these ideal elements at the theoretical level, and not with a great variety of radio-device structural elements differing in their characteristics, principles of operation, designs, etc. To apply the mathematical apparatus, further idealization is required; each of the above elements can be considered to be an active or passive one-port.

The methodological investigation of the history of science is very important to understand a methodology of the new scientific fields.

\section{REFERENCES}

Ahmed, H. (1991). Nanostructure fabrication. Proceedings of the IEEE, 79, 8. doi:10.1109/5.92073

Alù, A., \& Engheta, N. (2008). A Hertzian plasmonic nanodimer as an efficient optical nanoantenna. Physical Review B, 78, 19.

$\mathrm{http} / /$ repository.upenn.edu/cgi/viewcontent.cgi?article=1500\&conte $\mathrm{xt}=$ ese papers

doi:10.1103/PhysRevB.78.195111

Alù, A., Salandrino, A. \& Engheta, N. (2007). Coupling of optical lumped nanocircuit elements and effects. Optics Express, 15, 21. http://repository.upenn.edu/cgi/viewcontent.cgi?article=1477\& conte $\mathrm{xt}=$ ese $\_$papers doi:10.1364/OE.15.013865

Alù, A., Youngy, M. E., \& Engheta, N. (2008). Design of nanofilters for optical nanocircuits. Physical Review Letters B, 77, 144107. http://repository.upenn.edu/cgi/viewcontent.cgi?article=1436\&conte $\mathrm{xt}=\mathrm{ese}$ papers

Arnall, A. H. (2003). Future technologies, today's choices nanotechnology, artificial intelligence and robotics; a technical, political and institutional map of emerging technologies. A Report for the Greenpeace Environmental Trust, London: Department of Environmental Science and Technology Environmental Policy and Management Group, Faculty of Life Sciences, Imperial College London, University of London.

http://www.greenpeace.org.uk/MultimediaFiles/Live/FullReport/588 6.pdf

Barrett, D. (2000-2002). Radar theory. http://www.radarpages.co.uk/theory/ap3302/sec1/sec1 contents.htm

Berger, M. (2007). Towards wet computing. Nanowerk $L$.

http://www.nanowerk.com/spotlight/spotid=3559.php

Bernstein, G. H., Chua, L. O., Csurgay, A. I. et al. (2006). Biologicallyinspired cellural machine architectures. In W. S. Bainbrige, \& M. C. Roco (Eds.), Managing nano-bio-infocogno innovations: Technologies in society. National Science and Technology Council's Subcommittee on Nanoscale Science, Engineering, and Technology. Dordrecht: Springer.

Bhushan, B. (Ed.) (2004). Springer handbook of nanotechnology. Berlin, Heidelberg, New York: Springer-Verlag. doi:10.1007/3-540-29838-X

Burke, P. J., Li, S., \& Yu, Z. (2004). Quantitative theory of nanowire and nanotube antenna performance. http://arxiv.org/PS_cache/cond-mat/pdf/0408/0408418v1.pdf

Cox, D. M. (1999). High surface area materials. In R. W. Siegel, E. Hu, \& M. C. Roco (Eds.), Nanostructure science and technology. A worldwide study. $R$ \& D status and trends in nanoparticles, nanostructured materials, and nanodevices. Final Report Prepared under the Guidance of the Interagency Working Group on NanoScience, Engineering and Technology (IWGN), National Science and Technology Council (NSTC). Maryland: WTEC, Loyola College. http://www.wtec.org/loyola/nano/final/ch4.pdf

Engheta, N., Salandrino, A., \& Aiu, A. (2004). Circuit elements at optical frequencies: nano-inductors, nano-capacitors and nano-resistors. http://arxiv.org/ftp/cond-mat/papers/0411/0411463.pdf

Gorokhov, V. (2006). Die karlsruher experimente von heinrich hertz und die rolle ferdinand brauns für die entstehung der radiotechnik als theorie und praxis in Deutschland und in Russland. In Jahrbuch des Deutsch-Russischen kollegs 2004/2005. Aachen: Shaker Verlag GmbH.

Johnson, R. C. (2005). Nanotubes enable dense supercapacitors. http://www.automotivedesignline.com/showArticle.jhtml?printableA rticle $=$ true \&article $I d=60405658$ )

Józsa, C., Tombros, N., Popinciuc, M., Jonkman, H. T., \& van Wees, B. J. (2008). Graphene spintronics-Injection and transport. In NIM workshop "interactions in hybrid nanosystems", Frauenwörth.

Leider, P., \& Klier, J. (2008). Electron transport in nanostructures on helium films. In Th. Schimmel et al. (Eds.), Nanotechnology - Physics, chemistry, and biology of functional nanostructures: Results of the first research programme Kompetenznetz "Funktionelle Nanostrukturen" (Competence Network on Functional Nanostructures). Stuttgart: Landesstiftung Baden-Württemberg.

Pool Jr., Ch. P., \& Owens, F. J. (2003). Introduction to nanotechnology. Hoboken, NJ: John Wiley \& Sons.

Ray, D. L. (2008). The end of the silicon era? Carbon nanotubes, the next great leap. http://www.nanowerk.com/spotlight/spotid=5706.php

Reuleaux, F. (1875). Kinematics of machinery outlines of a theory of machines. London: Macmillan and Co.

Roth, S., \& Kern, D. (2008). Self-assambly of carbon nanotube transistors. In Th. Schimmel et al. (Eds.), Nanotechnology - Physics, chemistry, and biology of functional nanostructures: Results of the first research programme Kompetenznetz "Funktionelle Nanostrukturen" (Competence Network on Functional Nanostructures). Stuttgart: Landesstiftung Baden-Württemberg.

Salandrino, A., Alù, A., \& Engheta, N. (2007). Parallel, series, and intermediate interconnections of optical nanocircuit elements part 1: Analytical solution. http://arxiv.org/ftp/arxiv/papers/0707/0707.1002.pdf

Schiemann, G. (2005). Nanotechnology and nature. On two criteria for understanding their relationship. HYLE-International Journal for Philosophy of Chemistry, 11, 1. http://www.hyle.org

Schmid, G. et al. (2006). Nanotechnology. Assessment and perspectives. Berlin, Heidelberg: Springer

Sherrity, S., Wiedericky, H. D., Mukherjeey, B. K., \& Sayerz, M. (1997). An accurate equivalent circuit for the unloaded piezoelectric vibrator in the thickness mode. Journal of Physics D: Applied Physics, 30.

http://ext1.rmc.ca/academic/physics/ferroelectrics/Scanneddocument s/ferro6.pdf 


\section{GOROKHOV}

Silveirinha, M. G., Alù, A., Li, J., \& Engheta, N. (2007). Nanoinsulators and nanoconnectors for optical nanocircuits.

http://arxiv.org/ftp/cond-mat/papers/0703/0703600.pdf

Smalley, E. (2005). Nano bridge builds logic. The Latest Technology Research News.

http://www.trnmag.com/Stories/2005/012605/Nano_bridge_builds_1 ogic_012605.html

Song, L., Zhao, Y., Sun, L., \& Xie, S. (2008). Water filled in singlewalled carbon nanotubes. NIM Workshop "Interactions in Hybrid Nanosystems", Frauenwörth.

http://www.nano-initiative-munich.de/fileadmin/media/events/Book of Abstracts 28408 final klein.pdf

Steele B. (2005). Making the big step from electronics to photonics by modulating a beam of light with electricity.
http://www.news.cornell.edu/stories/May05/LipsonElectroOptical.ws .html

Starzyk, J. A., \& Sliwa, E. (1984). Upward topological analysis of large circuits using directed graph representation. IEEE Transactions on Circuits and Systems, CAS-31, 4.

Wilson, B. (2007). Distributed parameters. http://cnx.org/content/m1043/latest/

Xie, F.-Q., Obermair, Ch., \& Schimmel, Th. (2007). Configuring a bistable atomic switch by repeated electrochemical cycling. V International Conference on Microelectronics and Computer Science. Nanoscale Phenomena-Fundamentals and Applications, "NANO2007”, Kishinev. 